\title{
A QUANTITATIVE STUDY OF THE PRENATAL CHANGES IN ANGULATION OF THE SPINAL NERVES
}

ALEXANDER BARRY

Department of Anatomy, University of Michigan, Ann Arbor

SEVEN FIGURES

The University of Michigan Embryological Collection has recently acquired several specimens exhibiting stages in the genesis of a variety of malformations of the nervous system. The study of these abnormalities has stimulated a restudy of certain aspects of normal development. One phase in particular in which the need for more accurate data has been repeatedly encountered is the angulation of the spinal nerves. In such malformations as myeloschisis, the spinal cord is so anchored to adjacent body structures that no cauda equina is formed (Patten, '53). Cephalic to such defects there is abnormal traction on the cord. One means of assessing the extent of this traction is to study the degree to which spinal nerves depart from normal angulation. Again, in connection with the Arnold-Chiari malformation of the rhombencephalon the angulation of the spinal nerves offers important evidence on the moot question of cord traction as a possible etiological factor. Still another instance in which disturbances of the angulation of the spinal nerves are known to occur are tumors of the spinal cord, especially those originating early in development. There is, therefore, a real need for detailed information on the normal changes in the angulation of the entire series of the spinal nerves during the course of development. This need is especially urgent since the best studies in this general field at present available are those of 
Kunitomo ('18) and Streeter ('19), which deal only with the changes in the lumbo-sacral region, and are primarily concerned with the formation of the cauda equina and the filum terminale.

The work here reported was undertaken in an attempt to fill this gap in our available information. It presents in quantitative terms the different degrees of angulation which the spinal nerves exhibit at different stages of human prenatal development. It has seemed most useful to publish it as a separate study of normal development. Any conclusions that may be drawn from its data in the interpretation of abnormal conditions may best be discussed separately in connection with specific anomalies.

TABLE 1

\begin{tabular}{lrrrrrrrrrrr}
\hline $\begin{array}{l}\text { Coll. No. } \\
\text { CR length }\end{array}$ & 459 & 668 & 302 & 472 & 211 & 686 & 316 & 477 & 137 & 136 \\
$\quad$ in millimeters & 19 & 36 & 46 & 72 & 95 & 114 & 130 & 155 & 175 & 345 \\
Age in weeks & 7 & $8+$ & $9+$ & $11+$ & 13 & $14+$ & 16 & 18 & 19 & term \\
\hline
\end{tabular}

\section{MATERIALS AND METHODS}

A well graded developmental series of 10 human embryos was carefully selected for normality (table 1). Subsequent study of these embryos did not bring to light any unsuspected abnormalities, and the quantitative changes found in this series with age showed a consistently progressive character. In addition these specimens checked well with those reported by other investigators (Sensenig, '51; Kunitomo, '18; Streeter, '19). These factors made it seem unnecessary to expend either the time or the material which would have been needed to expand this initial series of 10 specimens.

The two youngest embryos were studied by means of graphic reconstructions made from sagittal serial sections. The 8 older embryos were dissected to expose the length of the vertebral canal, and the entire series of spinal nerves and ganglia. The basic problem in mensuration involved the 
establishing of points of demarcation that could be determined with precision at all levels and at all ages. For dealing with the vertebral column the caudal margin of the pedicles of the vertebrae, cut at a plane determined by the level of the spinal nerve and ganglion, was chosen as more likely to yield consistent results than an attempt to fix the center

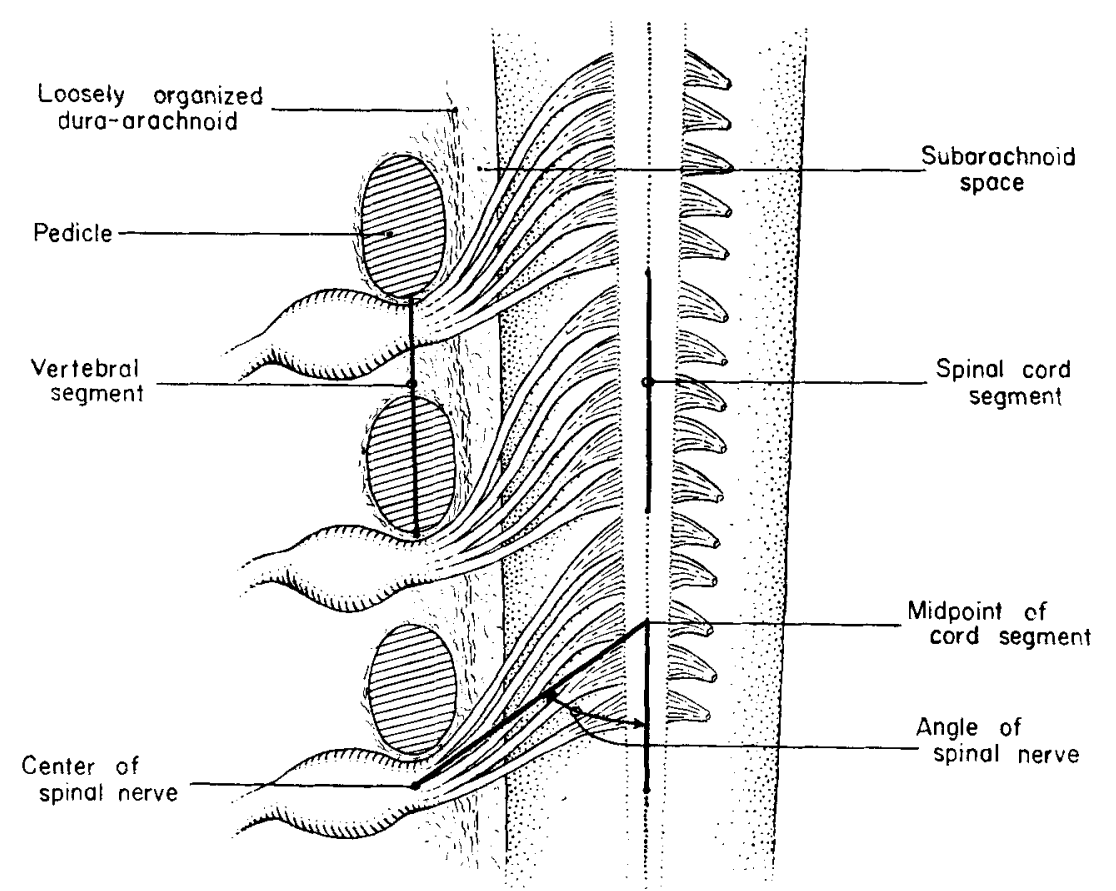

Fig. 1 Diagram slowing landmarks used to delimit a vertebral segment, a spinal cord segment, and the angle of a spinal nerve.

of either the pedicle or the intervertebral foramen. The distance between such points on adjacent vertebrae was taken as the length of that vertebral segment (fig. 1). For dealing with the spinal cord the points of emergence of the dorsal rootlets of the spinal nerves furnished the most sharply defined landmark. Their extent was taken as delimiting the length of the spinal cord segments. Accordingly, with the aid of a dissecting microscope, fine black glass needles were in- 
serted at the points between the caudalmost rootlet of one, and the most cephalic rootlet of the next spinal nerve. The distance between a pair of such markers was taken as the length of a spinal cord segment (fig. 1). These lengths were measured to the nearest tenth of a millimeter by means of a vernier caliper. Such measurements are of basic significance because they furnish information on the differential growth which underlies the angulation of the nerves.

The angulation of a spinal nerve could be measured directly in the following way. A dissecting microscope was adjusted so that its optical axis was perpendicular to the spinal cord at the level to be measured. One ocular contained a disc with an etched line of reference. This ocular was first rotated so that the line of reference lay parallel to the median dorsal sulcus of the spinal cord. It was then rotated to be in alignment with the spinal nerve. The angular difference between these positions could then be measured by means of a pointer extending radially from the ocular to the scale of a protractor. In many cases the spinal nerves did not run straight, but exhibited a curved course (fig. 1). The amount of such departure from the straight varied with age and level, due in large measure to the varying degrees of development of the dura and the degree of angulation of the spinal nerve. The course of a spinal nerve was defined arbitrarily as a line passing through two points, (a) the cephalocaudal midpoint of the spinal cord segment in the sagittal plane, and (b) the middle of the spinal nerve at the intervertebral foramen (fig. 1).

The method of direct measurement was satisfactory when the angulation of the nerve was not great. When the angle was acute, however, this means of determination led to considerable error. For this reason, in the later stages of the work, the angulation was usually determined indirectly. In this procedure use was made of the same two points described above as establishing the course of a spinal nerve. The cephalo-caudal distance (D) between these two points may be regarded as an index of the differential growth of the 
spinal cord and the vertebral column insofar as it has affected that spinal nerve (fig. 2). The lateral distance between the intervertebral foramen and the median dorsal sulcus was also measured and designated W. From these two measurements it was possible to calculate the angle of the spinal nerve, since the ratio of $D$ to $W$ is the cotangent of that angle.

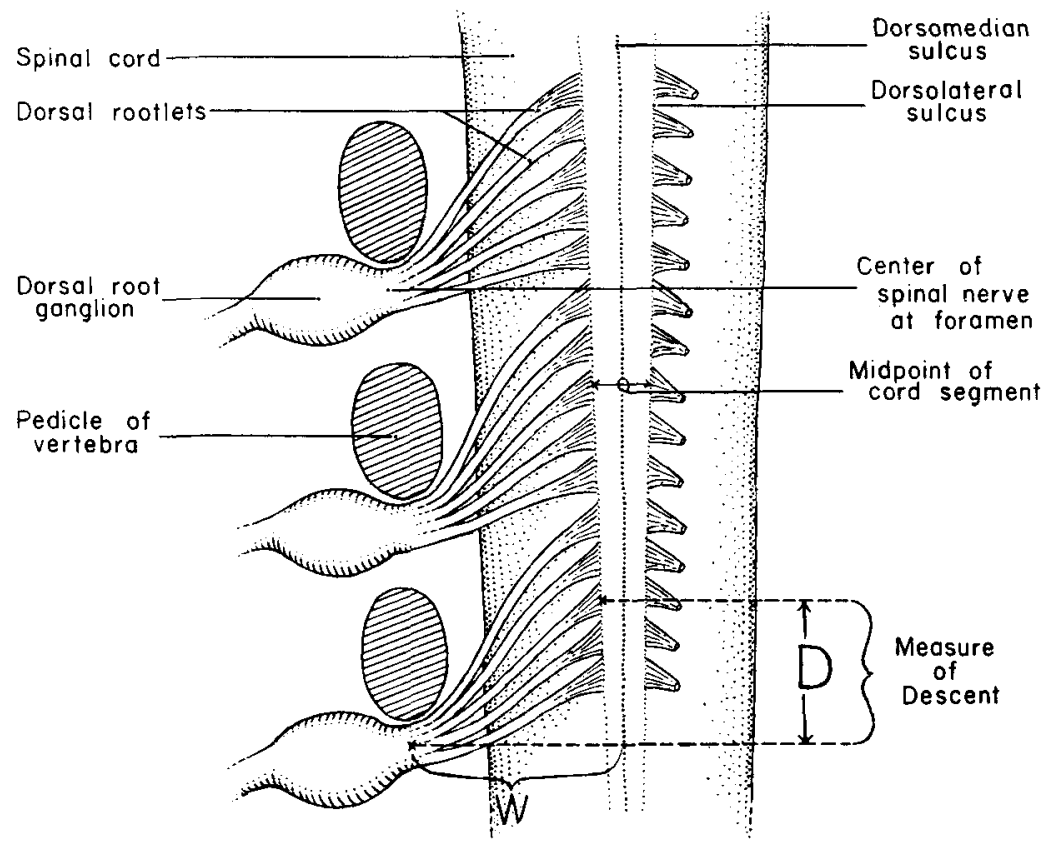

Fig. 2 Diagram showing landmarks used to determine the measure of descent of a spinal nerve (D), and the distance between the midline of the spinal cord and the intervertebral foramen (W). The ratio of $\mathrm{D}$ to $\mathrm{W}$ is the cotangent of the angle of the spinal nerve.

\section{RESULTS}

As Streeter phrased it ('19, p. 3), "In the younger stages the spinal cord and the vertebral column lie along side of each other in a metameric manner corresponding in position segment for segment." Thus in the earliest stages, when the spinal nerves have just been established each courses directly from its proper segment to its corresponding somite, running 
essentially at right angles to the sagittal plane of the body. This condition, however, is short-lived since the adjacent body segments increase in length more rapidly than do the spinal cord segments. By the 6th week (10 mm CR length), the sclerotomes have differentiated to a point where it is possible to identify the precartilagenous pedicles of the vertebrae (Bardeen and Lewis, '01). From this time on one may speak of vertebral segments and consider the morphological effects of the relative growth rates of the spinal and the vertebral segments. The relatively greater increase in length of the vertebral as compared with the spinal segments will pull the spinal nerves caudally and cause them to diverge from their original right angle with respect to the body axis.

The mesenchyme lying within the newly established vertebral canal gradually differentiates into the dura and the arachnoid. By about the 10 th to the 12 th week of development the dura has begun to attain its typical character as a tough fibrous membrane surrounding a distinct subarachnoid space (Sensenig, '51). Thereafter the angulation of the spinal nerves is seen within the subarachnoid space, since the dura is firmly adherent to the developing vertebrae.

The difference in the growth rate between the vertebral column and the spinal cord as a whole is a crucial factor in the attenuation of the caudalmost segments of the spinal cord to form the filum terminale (Kunitomo, '18; and Streeter, '19). The results obtained from the embryos used for this study corroborate and extend the work of these investigators (fig. 3). As can be seen, the caudal tip of the spinal cord extends to the tip of the coccyx throughout the first two months of development. At a stage of about $30 \mathrm{~mm}$ CR length (9th week) the relatively more rapid growth of the vertebral column results in the conus medullaris moving cephalad, and drawing out behind it a filum terminale.

It might be expected from the above data that the spinal nerves would not be drawn into angulation before the stage of $30 \mathrm{~mm}$ CR. Such, however, is not the case. If the spinal nerves are examined in an embryo of $19 \mathrm{~mm}$, it is evident 
that at certain levels they depart from their original right angle course. This emphasizes the necessity of considering the differential growth between the spinal cord and the surrounding tissues segment by segment. When this was done it was found that even in the youngest embryo studied (19 $\mathrm{mm}$ ) the first three or four cervical nerves sloped distinctly caudally. The next 8 spinal nerves ran essentially at right angles to the sagittal plane. Clearly this localized angulation

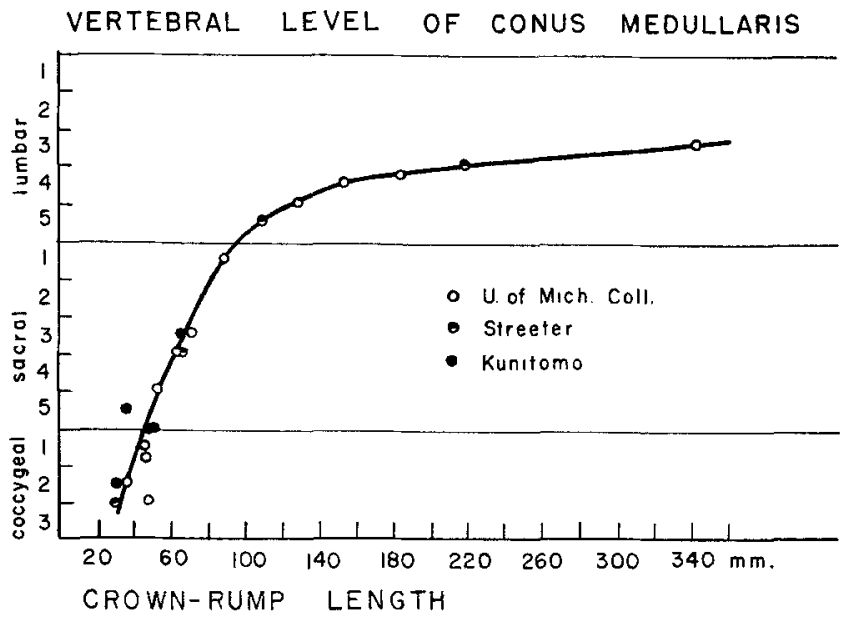

Fig. 3 Graph showing the position of the conus medullaris with respect to the bodies of the vertebrae at various stages of development. The data are from the work of Streeter and Kunitomo, combined with those from embryos used in this study.

of the high cervical nerves is not to be explained in terms of the overall growth of the spinal cord, but rather in terms of a local differential between the length of the cervical cord and the mesodermal structures of the upper neck. During the second month of development, one of the expressions of the different growth patterns in this region is a sharp increase in the nuchal flexure. This ventral bending results in an increase in length of the more dorsally located structures, such as the spinal cord. Since the cephalic end of the spinal cord is effectively anchored by the great mass of the brain, 
the stretching of the upper part of the cervical cord by the developing cervical flexure will tend to draw it cephalad. This inevitably causes the spinal nerves of this region to course caudally towards their foramina of exit. It should be noted that the effects of this stretching are dissipated within a relatively few segments, as is evidenced by the fact that the nerves caudal to the 4 th segment run in their original right-angle course with respect to the spinal cord.

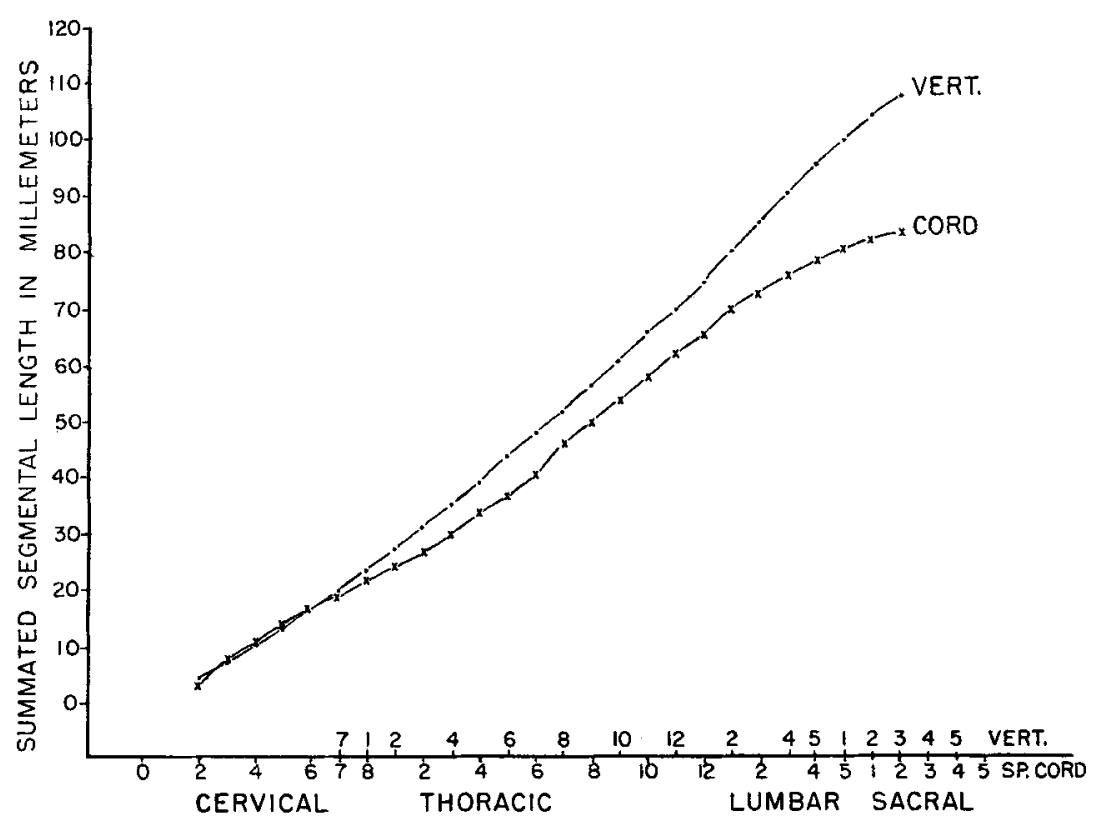

Fig. 4 Summative graphs showing the segmental lengths for both the vertebral column and the spinal cord of an embryo of 19 weeks. The points on these curves indicate the distance from the foramen magnum to the caudal edge of each segment.

The measurements of the lengths of the vertebral column segments and of the spinal cord segments can be presented in the form of a summative graph. Such a pair of curves is shown in figure 4 from an embryo of $175 \mathrm{~mm}$ CR. Since all the specimens yielded essentially the same types of curves, only this one is presented. These curves enable one to visualize, both for the spinal cord and for the vertebral column, 
the length of each segment as compared with the overall length of the structure. In addition, the differential length of the vertebral column and the spinal cord is indicated at each level by the distance between the curves. ${ }^{1}$ This differential length can be presented also either in terms of the angulation of the spinal nerves (fig. 5) or as the distance the nerve has been drawn caudally (figs. 6 and 7). From these graphs it is clear that the spinal cord can be divided into 4 regions according to the changes in angulation exhibited by the spinal nerves. It was unexpected to find that these regions did not correspond to the conventional morphological divisions of cervical, thoracic, lumbar, sacral, and coccygeal.

The first of these regions involves the upper 4 to 5 cervical segments. It can be seen that the degree of caudal slope becomes progressively less until at about the 5th cervical level the spinal nerve of the younger embryos has not been pulled from its original right angle course. This means that the upper 4 or 5 cervical spinal cord segments are longer than the corresponding vertebral segments. The second region includes the last 3 cervical and the first 4 thoracic segments. In this region the angle made by the spinal nerves with the spinal cord becomes progressively more acute, and the distance " $D$ " between the origin and emergence of the nerve becomes increasingly large. In this region, therefore, the vertebral segments are longer than the corresponding spinal cord segments. A third region extending from the 5th through the 10th thoracic segment, is one throughout which the spinal nerves have essentially the same degree of angulation with the body axis. This can only mean that throughout this region the vertebral and spinal cord segments are of corresponding lengths.

\footnotetext{
${ }^{1}$ In making such comparisons, the spinal cord segments were compared with the vertebral segments most closely aligned with them in their original orientation. Since there are 8 cervical spinal nerves, and only 7 cervical vertebrae, the first 7 spinal cord segments can be compared directly with the corresponding vertebrae. Caudal to this level, however, there is a one segment difference, e.g. the 8th cervical spinal cord segment is aligned with the first thoracic vertebral segment, etc.
} 
$46 \mathrm{~mm}$.

9 th wh.

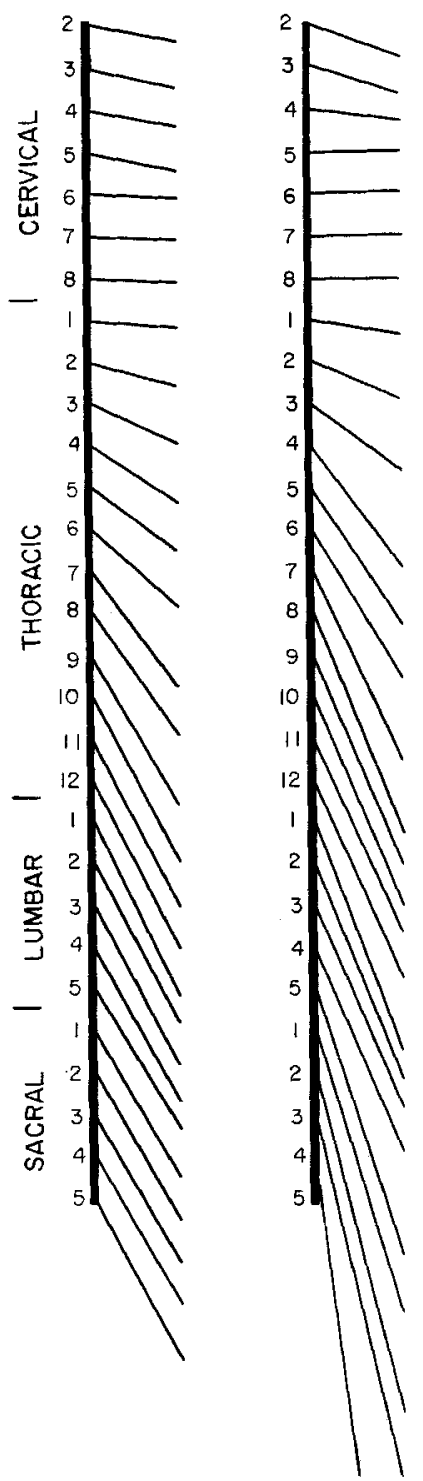

$130 \mathrm{~mm}$.

15th wk.

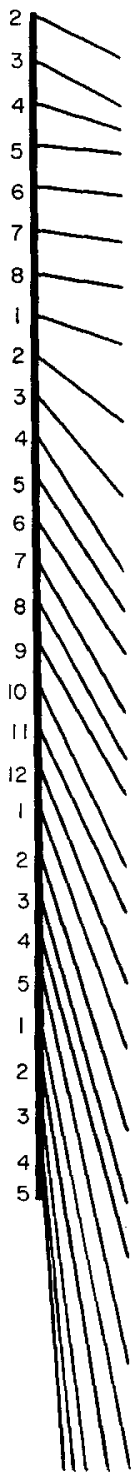

$175 \mathrm{~mm}$.

19 th wh.

Term

fetus
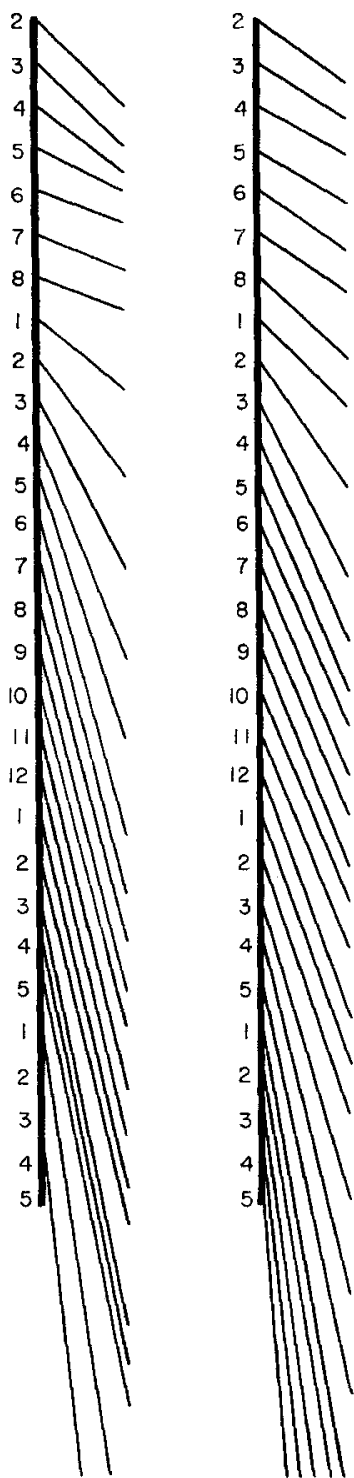

Fig. 5 Diagrammatic representation of the increasing acuity of angulation of the spinal nerves from the second cervical to the 5th sacral as they are found in embryos of 5 ages. 
Caudal to this level is a zone in which the younger embryos differ strikingly from the older ones with respect to the angulation of the spinal nerves. In this 4 th region, in the youngest embryo of this series ( $19 \mathrm{~mm} \mathrm{CR}$ ), it can be seen that the distance " $D$ " for the spinal nerves caudal to the 10th thoracic segment decreases until at levels caudal to the 4th lumbar it is zero. In other words the spinal nerves caudal to the 10th thoracic show a progressively less acute angulation to the spinal cord until caudal to the 4th lumbar segment they run

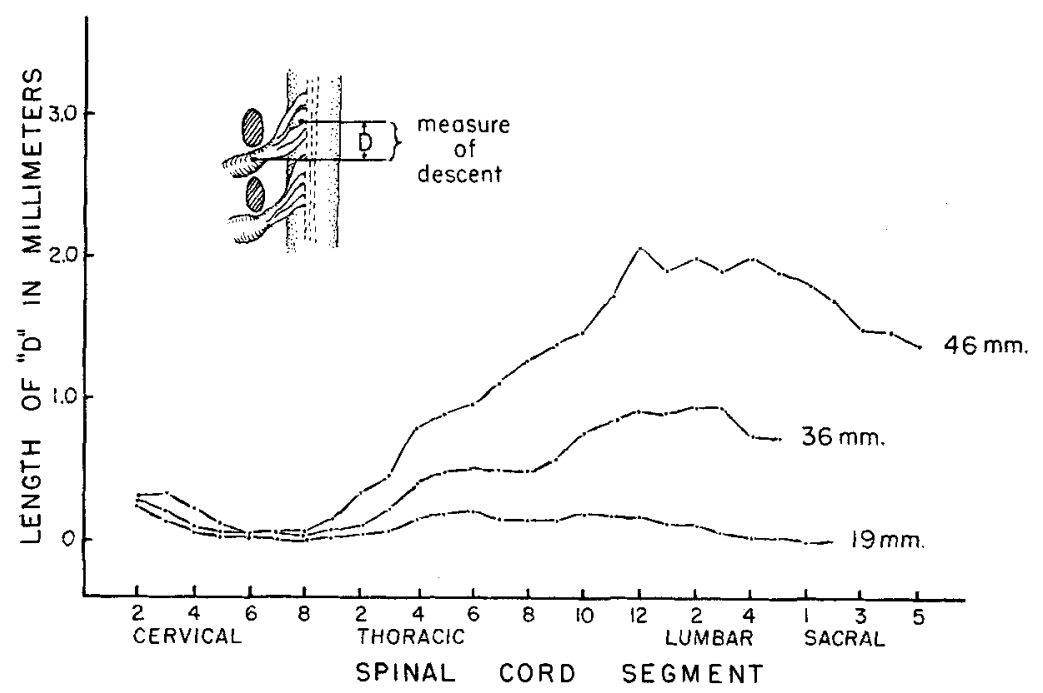

Fig. 6 Graphs showing, for embryos of 19,36 , and $46 \mathrm{~mm}$ CR, the amount of the descent of the spinal nerves at different segmental levels of the spinal cord.

at right angles. This is the consequence of the fact that in embryos so young that the spinal cord extends to the tip of the vertebral canal, the most caudal spinal nerves must course at right angles, even though they may slope caudally at higher levels. For this reason, the spinal nerves in this 4th region (caudal to 10 thoracic) must show a transition from the angle of thoracic levels to the perpendicularity of sacral levels. However, as soon as the tip of the spinal cord ascends within the vertebral canal, even the low sacral spinal nerves will slope caudally. The higher the conus medullaris ascends, the 
more acutely will these caudal nerves be slanted. This is borne out by the data from embryos of 36 and $46 \mathrm{~mm} \mathrm{CR}$ (fig. 6). In the older of these specimens the 5th sacral nerve has been drawn tailward so that it slopes caudally almost as much as the thoracic nerves. By the stage of $72 \mathrm{~mm} \mathrm{CR}$ (fig. 7) the low sacral nerves have been drawn to such an

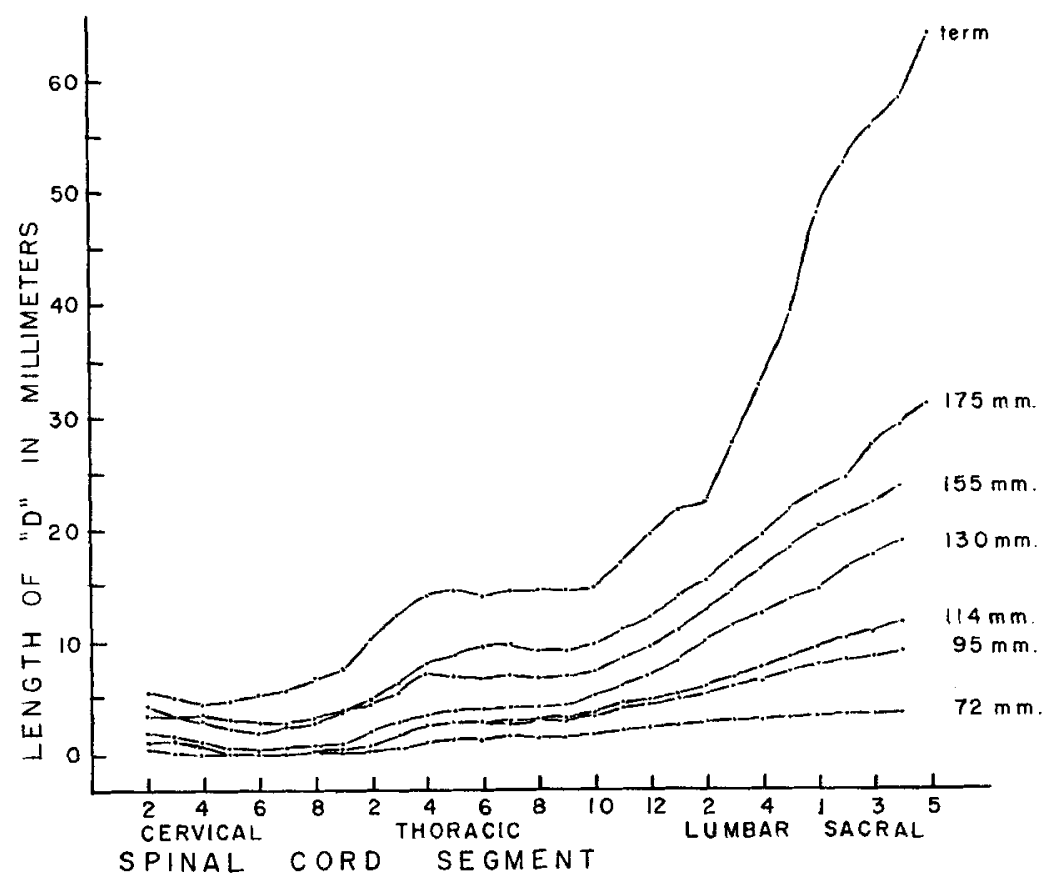

Fig. 7 Graphs showing for a series of 5 older embryos similar data to those presented in figure 6.

acute slope that the series of nerves of this 4 th zone shows a progressive increase in slant as one passes caudally. The curves from older specimens show that this situation becomes more striking with increasing age.

These facts are a striking demonstration of the phenomenon of cephalic precocity with respect to the vertebral column. The upper and middle thoracic vertebrae show an increase in length over the spinal cord segments by the 8th week 
$(36 \mathrm{~mm})$ of development, while the lumbosacral region does not exhibit this differential length until the 10th week.

\section{SUMMARY}

A graded series of 10 human embryos was studied with respect to the angulation of their spinal nerves. The angle made by each spinal nerve was measured directly, and also indirectly utilizing the distance each nerve had been drawn caudad by the pedicle of the corresponding vertebra. These results are presented graphically.

Analysis of these data shows that the spinal cord can be divided into 4 regions with respect to the angulation of the spinal nerves. The first region (upper 4 cervical) is one in which the caudal slant of the high cervical nerves becomes progressively less marked. Throughout the second region (5C through $4 \mathrm{~T}$ ) this trend is reversed, and the spinal nerves exhibit an increasing tailward slope. The spinal nerves of the third region (5T through $10 \mathrm{~T}$ ) show essentially no change in angulation. The 4th zone (caudal to $10 \mathrm{~T}$ ) lags behind the others in development so that in specimens younger than $72 \mathrm{~mm}$ CR $(11+$ wks.) the caudal slope of the spinal nerves becomes progressively less as one approaches the coccygeal segments. In older specimens, however, the rate of elongation of the vertebral column has overtaken that of this 4th zone of the spinal cord. As a result the tailward slope of the spinal nerves caudal to $10 \mathrm{~T}$ becomes progressively greater, with the resultant formation of the filum terminale and cauda equina.

\section{LITERATURE GITED}

Bardeen, C. R., AND W. H. Lewis 1901 The development of the limbs, body wall, and back. Am. J. Anat., 1: 1-37.

Kunitomo, K. 1918 The development and reduetion of the tail and of the caudal end of the spinal cord in the human embryo. Contrib. to Embryol. no. $26,8: 163-197$.

PATTEN, B. M. 1953 Embryological stages in the establishing of myelosehisis with spina bifida. Am. J. Anat., 93: 365-396. 
SEnsenia, E. C. 1951 The early development of the meninges of the spinal cord in human embryos. Contrib. to Embryol, no. 228, 34: 145-157.

Streeter, G. L. 1919 Factors involved in the formation of the filum terminale. Am. J. Anat., 25: 1-11. 